\title{
USO DE EQUAÇÕES LINEARES NA DETERMINAÇÃO DOS PARÂMETROS DE MICHAELIS-MENTEN
}

Nakédia M. F. Carvalho, Bianca M. Pires, Octavio A. C. Antunes ${ }^{\dagger}$ e Roberto B. Faria*

Instituto de Química, Universidade Federal do Rio de Janeiro, Av. Athos da Silveira Ramos, 149, CT Bl. A, 21941-909 Rio de Janeiro - RJ, Brasil

Renata E. H. M. B. Osório, Clovis Piovezan e Ademir Neves

Universidade Federal de Santa Catarina, Departamento de Química, Campus Trindade, CP 476, 88040-900 Florianópolis - SC, Brasil

Recebido em 4/11/09; aceito em 26/3/10; publicado na web em 20/7/10

\begin{abstract}
USE OF LINEAR EQUATIONS TO OBTAIN MICHAELIS-MENTEN PARAMETERS. The Michaelis-Menten equation is used in many biochemical and bioinorganic kinetic studies involving homogeneous catalysis. Otherwise, it is known that determination of Michaelis-Menten parameters $K_{\mathrm{M}}, V_{\max }$, and $k_{\text {cat }}$ by the well-known Lineweaver-Burk double reciprocal linear equation does not produce the best values for these parameters. In this paper we present a discussion on different linear equations which can be used to calculate these parameters and we compare their results with the values obtained by the more reliable nonlinear least-square fit.
\end{abstract}

Keywords: Michaelis-Menten; Lineweaver-Burk; nonlinear least-square fit.

\section{INTRODUÇÃO}

No estudo de reações enzimáticas e biomiméticas utiliza-se comumente o esquema de Briggs-Haldane, ${ }^{1}$ que considera as reações de formação e dissociação do complexo enzima-substrato, ES, e sua reação de decomposição formando os produtos e regenerando a enzima.

$$
\begin{aligned}
& \mathrm{S}+\mathrm{E} \stackrel{k_{1}}{\mathrm{ES} \stackrel{k_{-1}}{\longrightarrow}} \mathrm{ES} \\
& \mathrm{ES}+\mathrm{E}
\end{aligned}
$$

Aplicando-se a este esquema cinético a aproximação do estado estacionário (aproximação de Bodenstein) para a concentração do complexo enzima-substrato obtemos a famosa equação de hipérbole retangular de Michaelis-Menten para a velocidade inicial da reação (Equação 1). ${ }^{2-5}$

$$
\mathrm{v}_{0}=k_{2}[\mathrm{E}]_{0}[\mathrm{~S}]_{0} /\left([\mathrm{S}]_{0}+K_{\mathrm{M}}\right)=V_{\max }[\mathrm{S}]_{0} /\left([\mathrm{S}]_{0}+K_{\mathrm{M}}\right)
$$

onde, $K_{\mathrm{M}}=\left(k_{-1}+k_{2}\right) / k_{1}$ é a constante de Michaelis e $k_{2}[\mathrm{E}]_{0}=V_{\max }$ é a velocidade máxima da reação para uma dada concentração inicial da enzima, $[\mathrm{E}]_{0}$, na presença de um excesso de substrato. A constante de velocidade $k_{2}$ é também chamada de $k_{\text {cat }}$ ou número de renovação (turnover number, turnover frequency).

Para se obter $K_{\mathrm{M}}, V_{\max }$ e $k_{\text {cat }}$, o melhor procedimento é o uso de um ajuste não linear por mínimos quadrados (nonlinear least-square method) dos dados experimentais de $\mathrm{v}_{0}$ contra $[\mathrm{S}]_{0}{ }^{6}{ }^{6}$ Signorella et al. ${ }^{7}$ mostraram recentemente que $k_{\text {cat }}$ é especialmente sensível ao método de ajuste empregado, enfatizando que o ajuste não linear por mínimos quadrados é o mais confiável. Por outro lado, um ajuste linear menos confiável do ponto de vista estatístico pode ser feito usando-se a equação de duplo recíproco de Lineweaver-Burk (Equação 2), ${ }^{8}$ que pode ser obtida após manipulação da Equação 1.

$$
1 / \mathrm{v}_{0}=K_{\mathrm{M}} /\left(V_{\max }[\mathrm{S}]_{0}\right)+1 / V_{\max }
$$

*e-mail: faria@iq.ufrj.br

${ }^{\dagger}$ In Memoriam
Assim, um gráfico de $1 / \mathrm{v}_{0}$ contra $1 /[\mathrm{S}]_{0}$ permite a determinação de $1 / V_{\max }$ como a intercessão da reta com o eixo das ordenadas (coeficiente linear) e $K_{\mathrm{M}} / V_{\max }$ pela inclinação da reta (coeficiente angular), a partir dos quais $K_{\mathrm{M}}$ e $V_{\text {max }}$ podem ser obtidos.

Apesar do fato de que diversos pacotes computacionais atuais oferecem a possibilidade de um ajuste não linear por mínimos quadrados, um ajuste linear é mais simples de ser apresentado e compreendido pelos estudantes, bem como, geralmente, permite uma percepção mais imediata dos pontos experimentais que apresentem maiores desvios de um comportamento do tipo Michaelis-Menten (quando a maioria dos pontos não segue um ajuste linear, isto indica que podemos estar diante de outro tipo de mecanismo onde ocorram, como por exemplo, etapas de inibição enzimática, um aspecto que não será abordado no presente artigo). Entretanto, como é sabido que o uso da Equação 2 não produz valores confiáveis para os parâmetros de Michaelis-Menten, ${ }^{2,4,7}$ apresentamos neste trabalho uma discussão sobre quatro diferentes equações lineares que podem ser obtidas por simples manipulação da Equação 1, com o objetivo de escolher qual delas produz os melhores parâmetros. A abordagem que escolhemos para este estudo foi a de comparar os parâmetros de Michaelis-Menten obtidos por cada uma das equações lineares com aqueles obtidos pelo ajuste não linear por mínimos quadrados, que é o mais confiável. Os resultados obtidos mostram que duas dessas equações lineares, uma delas aqui apresentada pela primeira vez, podem ser utilizadas para o cálculo dos parâmetros de Michaelis-Menten, uma vez que os valores obtidos são muito próximos daqueles obtidos por ajuste não linear por mínimos quadrados e significativamente mais confiáveis do que os obtidos pela Equação de Lineweaver-Burk.

\section{MÉTODOS}

Os ajustes não lineares por mínimos quadrados dos dados experimentais foram feitos empregando-se o pacote computacional Origin 6.1. ${ }^{9}$ Os ajuste lineares (regressão linear) foram obtidos empregandose a planilha eletrônica Lotus 1-2-3. ${ }^{10}$

Os dados experimentais utilizados são provenientes de estudos cinéticos atualmente sendo feitos em nossos laboratórios envolvendo a síntese de compostos bioinorgânicos miméticos de enzimas naturais. O conjunto de dados 1 (ver Tabela 1) refere-se ao acompanhamento cinético da atividade de catalase (reação de decomposição do peró- 
xido de hidrogênio) de um complexo tetranuclear de ferro(III) com o ligante 1,3-bis[((2-aminoetil)amino]-2-propanol). Os conjuntos de dados 2 e 4 (ver Tabela 3) referem-se à hidrólise do 2,4-bis-dinitrofenilfostato e do 3,5-di-terc-butilcatecol por um complexo dinuclear de $\mathrm{Cu}$ e o conjunto de dados 3 refere-se à oxidação do 3,5-di-tercbutilcatecol catalisada por um complexo dinuclear de $\mathrm{Ni}$.

\section{RESULTADOS E DISCUSSÃO}

Diferentes equações lineares podem ser obtidas por meio de simples manipulações da Equação 1, dentre as quais temos as equações de Lineweaver-Burk (Equação 2), ${ }^{8}$ de Hanes (Equação 3$)^{11}$ e de EadieHofstee (Equação 4). ${ }^{12,13}$ A estas podemos acrescentar a Equação 5 que, no melhor do nosso conhecimento, ainda não foi investigada. A partir destas equações os parâmetros de Michaelis-Menten podem ser facilmente obtidos.

$$
\begin{gathered}
1 / \mathrm{v}_{0}=K_{\mathrm{M}} /\left(V_{\text {max }}[\mathrm{S}]_{0}\right)+1 / V_{\max } \\
{[\mathrm{S}]_{0} / \mathrm{v}_{0}=[\mathrm{S}]_{0} / V_{\text {max }}+K_{\mathrm{M}} / V_{\text {max }}} \\
\mathrm{v}_{0}=V_{\text {max }}-K_{\mathrm{M}}\left(\mathrm{v}_{0} /[\mathrm{S}]_{0}\right) \\
{[\mathrm{S}]_{0}=V_{\text {max }}[\mathrm{S}]_{0} / \mathrm{v}_{0}-K_{\mathrm{M}}}
\end{gathered}
$$

A Equação 2 permite a determinação de $V_{\max }$ e $K_{\mathrm{M}}$ a partir de um gráfico de $1 / \mathrm{v}_{0}$ contra $1 /[\mathrm{S}]_{0}$, a Equação 3 a partir de um gráfico de $[\mathrm{S}]_{0} / \mathrm{v}_{0}$ contra $[\mathrm{S}]_{0}$, a Equação 4 a partir de um gráfico de $\mathrm{v}_{0}$ contra $\mathrm{v}_{0} /[\mathrm{S}]_{0}$, e a Equação 5 a partir de um gráfico de $[\mathrm{S}]_{0}$ contra $[\mathrm{S}]_{0} / \mathrm{v}_{0}$. $\mathrm{O}$ uso das Equações 4 e 5 parece mais simples que as Equações 2 e 3 , uma vez que os coeficientes linear e angular das retas fornecem diretamente os valores de $V_{\max } \mathrm{e}-K_{\mathrm{M}}$.

A Equação de Hanes (3) foi investigada por Lineweaver e Burk ${ }^{8}$ que concluiram que a Equação 2 produzia melhores resultados para baixas concentrações de substrato. Entretanto, Wilkinson ${ }^{14}$ ao fazer uma análise estatística detalhada das Equações 2 e 3 concluiu que esta última era superior. A comparação estatística feita por Dowd e Riggs ${ }^{15}$ das Equações 2, 3 e 4 confirmou a inferioridade da Equação 2 e indicou a Equação 3 como uma melhor opção quando o erro em $\mathrm{v}_{0}$ era pequeno, mas recomendou a Equação 4 quando o erro em $\mathrm{v}_{0}$ fosse grande.
Embora uma análise estatística da confiabilidade das diferentes equações lineares empregadas para a determinação dos parâmetros de Michaelis-Menten seja relevante, ${ }^{16}$ a abordagem que apresentamos neste trabalho foi a de comparar os valores dos parâmetros obtidos pelas diferentes equações lineares com os obtidos pelo método mais confiável do ajuste não linear por mínimos quadrados. ${ }^{6,7}$

A Tabela 1 mostra os parâmetros de Michaelis-Menten calculados a partir dos dados experimentais de velocidade inicial (Conjunto 1) para a atividade de catalase (reação de decomposição do peróxido de hidrogênio) de um complexo de ferro(III). Os valores experimentais de velocidade inicial empregados são mostrados na Tabela 2, juntamente com os valores de $\mathrm{v}_{0}$ calculados pela Equação 1 usando-se os parâmetros de Michaelis-Menten obtidos tanto pelo método de ajuste não linear por mínimos quadrados quanto pelas Equações 2 a 5. A Figura 1 apresenta uma comparação gráfica dos valores de $\mathrm{v}_{0}$ obtidos.

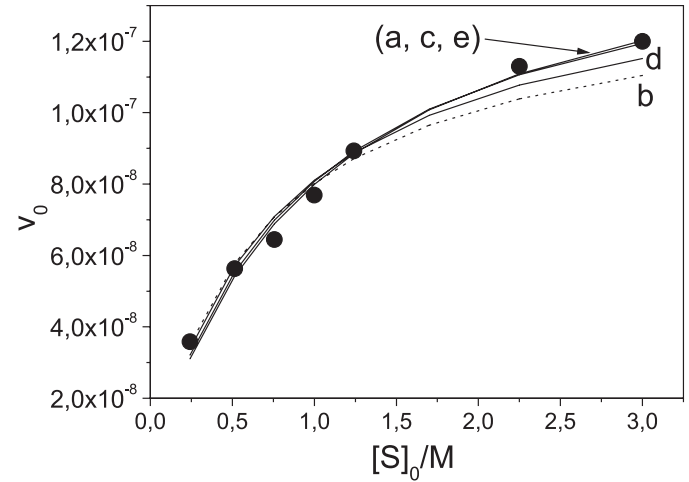

Figura 1. Valores experimentais $(\bullet)$ e calculados (linhas contínuas e pontilhadas) de $v_{0}$ para o conjunto de dados 1 , usando a Equação 1 e os parâmetros obtidos por: a) ajuste não linear por mínimos quadrados, b) Equação 2, Lineweaver-Burk (linha pontilhada), c) Equação 3, Hanes, d) Equação 4, Eadie-Hofstee, e) Equação 5. Ver Tabela 2 para os valores numéricos

Como pode ser visto na Tabela 1, é boa a concordância entre os parâmetros obtidos pelo método de ajuste não linear por mínimos quadrados e as Equações 3 e 5, diferentemente dos valores obtidos pelas Equações de Lineweaver-Burk e Eadie-Hofstee (2 e 4, respectivamente). Os parâmetros obtidos pelo uso das Equações 2 e 4, quando

Tabela 1. Parâmetros de Michaelis-Menten determinados por ajuste não linear por mínimos quadrados e pelas Equações 2 a 5 para o conjunto de dados 1 , atividade de catalase de um complexo tetranuclear de ferro(III) (veja Tabela 2 e Figura 1). [E] $]_{0}=1,25 \times 10^{-3} \mathrm{M}$

\begin{tabular}{|c|c|c|c|c|c|c|}
\hline \multirow[b]{2}{*}[\mathrm{S}]{$_{0} / \mathrm{M}$} & \multicolumn{6}{|c|}{$\mathrm{v}_{0} / 10^{-8} \mathrm{M} \mathrm{s}^{-1}$} \\
\hline & Experimental & $\begin{array}{l}\text { Ajuste não linear por } \\
\text { mínimos quadrados }\end{array}$ & $\begin{array}{c}\text { Lineweaver-Burk } \\
\text { Equação } 2 \\
\end{array}$ & $\begin{array}{c}\text { Hanes } \\
\text { Equação } 3\end{array}$ & $\begin{array}{c}\text { Eadie-Hofstee } \\
\text { Equação } 4\end{array}$ & Equação 5 \\
\hline 0,2421 & 3,585 & 3,106 & 3,510 & 3,182 & 3,389 & 3,206 \\
\hline 0,5144 & 5,630 & 5,422 & 5,780 & 5,514 & 5,710 & 5,539 \\
\hline 0,7565 & 6,445 & 6,881 & 7,083 & 6,968 & 7,092 & 6,986 \\
\hline 0,9986 & 7,695 & 7,988 & 8,014 & 8,062 & 8,102 & 8,072 \\
\hline 1,241 & 8,930 & 8,857 & 8,712 & 8,916 & 8,872 & 8,918 \\
\hline 2,250 & 11,30 & 11,09 & 10,39 & 11,09 & 10,77 & 11,06 \\
\hline 3,000 & 12,00 & 12,02 & 11,04 & 11,99 & 11,52 & 11,94 \\
\hline
\end{tabular}

\begin{tabular}{cccccc}
\hline & $\begin{array}{c}\text { Ajuste não linear por míni- } \\
\text { mos quadrados }\end{array}$ & $\begin{array}{c}\text { Lineweaver-Burk, } \\
\text { Equação 2 }\end{array}$ & Hanes, Equação 3 & Eadie-Hofstee, Equação 4 & Equação 5 \\
\hline$K_{\mathrm{M}}$ & $1,01 \pm 0,11$ & $0,696 \pm 0,124$ & $0,963 \pm 0,141$ & $0,800 \pm 0,099$ & $0,944 \pm 0,101$ \\
$V_{\max } / 10^{-7}$ & $1,61 \pm 0,09$ & $1,36 \pm 0,17$ & $1,58 \pm 0,07$ & $1,46 \pm 0,09$ & $1,57 \pm 0,07$ \\
$k_{\mathrm{cal}} / 10^{-4}$ & $1,29 \pm 0,06$ & $1,09 \pm 0,13$ & $1,27 \pm 0,05$ & $1,17 \pm 0,06$ & $1,26 \pm 0,05$ \\
\hline
\end{tabular}

Tabela 2. Valores experimentais de velocidade inicial para o conjunto de dados 1 e valores de $\mathrm{v}_{0}$ calculados pela Equação 1 usando-se os parâmetros de MichaelisMenten obtidos por ajuste não linear por mínimos quadrados e pelas Equações 2 a 5 (veja Figura 1). [E $]_{0}=1,25 \times 10^{-3} \mathrm{M}$ 
Tabela 3. Parâmetros de Michaelis-Menten determinados usando-se ajuste não linear por mínimos quadrados e as Equações 2 a 5 para os conjuntos de dados 2 a 4

\begin{tabular}{|c|c|c|c|c|c|}
\hline & $\begin{array}{l}\text { Ajuste não linear por } \\
\text { mínimos quadrados }\end{array}$ & $\begin{array}{c}\text { Lineweaver-Burk, } \\
\text { Equação } 2\end{array}$ & $\begin{array}{c}\text { Hanes, } \\
\text { Equação } 3\end{array}$ & $\begin{array}{l}\text { Eadie-Hofstee, } \\
\text { Equação } 4\end{array}$ & Equação 5 \\
\hline & \multicolumn{5}{|c|}{ Conjunto de dados 2: hidrólise do 2,4-bis-dinitro-fenilfosfato por um complexo dinuclear de $\mathrm{Cu}$. $[\mathrm{E}]_{0}=4,0 \times 10^{-5} \mathrm{M}$} \\
\hline$K_{\mathrm{M}} / 10^{-3}$ & $3,82 \pm 0,47$ & $5,27 \pm 0,88$ & $4,05 \pm 0,57$ & $4,33 \pm 0,53$ & $3,93 \pm 0,34$ \\
\hline$V_{\max } / 10^{-8}$ & $1,20 \pm 0,06$ & $1,41 \pm 0,18$ & $1,22 \pm 0,07$ & $1,26 \pm 0,06$ & $1,21 \pm 0,07$ \\
\hline \multirow[t]{2}{*}{$k_{\mathrm{cat}} / 10^{-4}$} & $3,00 \pm 0,16$ & $3,52 \pm 0,45$ & $3,06 \pm 0,17$ & $3,16 \pm 0,14$ & $3,01 \pm 0,16$ \\
\hline & \multicolumn{5}{|c|}{ Conjunto de dados 3: hidrólise do 2,4-bis-dinitro-fenilfosfato por um complexo dinuclear de Ni. $[\mathrm{E}]_{0}=1,0 \times 10^{-5} \mathrm{M}$} \\
\hline$K_{\mathrm{M}} / 10^{-4}$ & $8,30 \pm 1,90$ & $13,03 \pm 2,45$ & $8,96 \pm 0,23$ & $10,13 \pm 1,83$ & $7,93 \pm 1,37$ \\
\hline$V_{\max } / 10^{-7}$ & $3,62 \pm 0,37$ & $4,58 \pm 0,72$ & $3,69 \pm 0,37$ & $3,95 \pm 0,28$ & $3,48 \pm 0,35$ \\
\hline \multirow[t]{2}{*}{$k_{\mathrm{cat}} / 10^{-2}$} & $3,62 \pm 0,37$ & $4,58 \pm 0,72$ & $3,69 \pm 0,37$ & $3,95 \pm 0,28$ & $3,48 \pm 0,35$ \\
\hline & \multicolumn{5}{|c|}{ Conjunto de dados 4: oxidação do 3,5-di-terc-butilcatecol por um complexo dinuclear de $\mathrm{Cu}$. $[\mathrm{E}]_{0}=2,4 \times 10^{-5} \mathrm{M}$} \\
\hline$K_{\mathrm{M}} / 10^{-3}$ & $3,47 \pm 0,48$ & $2,67 \pm 0,29$ & $3,52 \pm 0,59$ & $2,85 \pm 0,30$ & $3,39 \pm 0,42$ \\
\hline$V_{\max } / 10^{-7}$ & $6,18 \pm 0,32$ & $5,59 \pm 0,39$ & $6,23 \pm 0,31$ & $5,76 \pm 0,27$ & $6,14 \pm 0,30$ \\
\hline$k_{\mathrm{cat}} / 10^{-2}$ & $2,57 \pm 0,14$ & $2,33 \pm 0,16$ & $2,60 \pm 0,13$ & $2,40 \pm 0,11$ & $2,56 \pm 0,13$ \\
\hline
\end{tabular}

Tabela 4. Valores experimentais de velocidade inicial para o conjunto de dados 2 e valores de $\mathrm{v}_{0}$ calculados pela Equação 1 usando-se os parâmetros de MichaelisMenten obtidos por ajuste não linear por mínimos quadrados e pelas Equações 2 a 5 (veja Tabela 3 e Figura 2). [E $]_{0}=4,0 \times 10^{-5} \mathrm{M}$

\begin{tabular}{|c|c|c|c|c|c|c|}
\hline \multirow[b]{2}{*}[\mathrm{S}]{$_{0} / \mathrm{M}$} & \multicolumn{6}{|c|}{$\mathrm{v}_{0} / 10^{-9} \mathrm{M} \mathrm{s}^{-1}$} \\
\hline & Experimental & $\begin{array}{l}\text { Ajuste não linear por } \\
\text { mínimos quadrados }\end{array}$ & $\begin{array}{c}\text { Lineweaver-Burk, } \\
\text { Equação } 2\end{array}$ & $\begin{array}{c}\text { Hanes, } \\
\text { Equação } 3\end{array}$ & $\begin{array}{l}\text { Eadie-Hofstee, } \\
\text { Equação } 4\end{array}$ & Equação 5 \\
\hline 0,0012 & 2,56322 & 2,866 & 2,610 & 2,793 & 2,741 & 2,822 \\
\hline 0,0024 & 4,68402 & 4,626 & 4,403 & 4,547 & 4,504 & 4,573 \\
\hline 0,0036 & 5,82961 & 5,816 & 5,711 & 5,752 & 5,734 & 5,765 \\
\hline 0,0048 & 7,00386 & 6,675 & 6,707 & 6,630 & 6,641 & 6,630 \\
\hline 0,0060 & 7,31873 & 7,325 & 7,491 & 7,298 & 7,338 & 7,285 \\
\hline 0,0072 & 7,82369 & 7,832 & 8,125 & 7,824 & 7,889 & 7,799 \\
\hline 0,0084 & 8,04077 & 8,241 & 8,647 & 8,248 & 8,336 & 8,213 \\
\hline
\end{tabular}

Tabela 5. Valores experimentais de velocidade inicial para o conjunto de dados 3 e valores de $\mathrm{v}_{0}$ calculados pela Equação 1 usando-se os parâmetros de MichaelisMenten obtidos por ajuste não linear por mínimos quadrados e pelas Equações 2 a 5 (veja Tabela 3 e Figura 3). [E] $]_{0}=1,0 \times 10^{-5} \mathrm{M}$

\begin{tabular}{|c|c|c|c|c|c|c|}
\hline \multirow[b]{2}{*}[\mathrm{S}]{$_{0} / 10^{-4} \mathrm{M}$} & \multicolumn{6}{|c|}{$\mathrm{v}_{0} / 10^{-7} \mathrm{M} \mathrm{s}^{-1}$} \\
\hline & Experimental & Nonlinear least-square & $\begin{array}{c}\text { Lineweaver-Burk, } \\
\text { Equação } 2\end{array}$ & $\begin{array}{c}\text { Hanes, } \\
\text { Equação } 3\end{array}$ & $\begin{array}{l}\text { Eadie-Hofstee, } \\
\text { Equação } 4\end{array}$ & Equação 5 \\
\hline 2,0 & 0,605096 & 0,7028 & 0,6099 & 0,6739 & 0,6507 & 0,7017 \\
\hline 4,0 & 1,08402 & 1,177 & 1,076 & 1,140 & 1,117 & 1,168 \\
\hline 6,0 & 1,45455 & 1,519 & 1,445 & 1,481 & 1,486 & 1,501 \\
\hline 8,0 & 1,85565 & 1,776 & 1,743 & 1,742 & 1,741 & 1,750 \\
\hline 10 & 2,13912 & 1,978 & 1,990 & 1,948 & 1,961 & 1,943 \\
\hline 12 & 2,24848 & 2,140 & 2,197 & 2,114 & 2,140 & 2,098 \\
\hline 14 & 2,27851 & 2,272 & 2,374 & 2,252 & 2,290 & 2,224 \\
\hline 18 & 2,28967 & 2,477 & 2,659 & 2,466 & 2,525 & 2,418 \\
\hline
\end{tabular}

Tabela 6. Valores experimentais de velocidade inicial para o conjunto de dados 4 e valores de $\mathrm{v}_{0}$ calculados pela Equação 1 usando-se os parâmetros de MichaelisMenten obtidos por ajuste não linear por mínimos quadrados e pelas Equações 2 a 5 (veja Tabela 3 e Figura 4). [E] $]_{0}=2,4 \times 10^{-5} \mathrm{M}$

\begin{tabular}{|c|c|c|c|c|c|c|}
\hline \multirow[b]{2}{*}[\mathrm{S}]{$_{0} / 10^{-3} \mathrm{M}$} & \multicolumn{6}{|c|}{$\mathrm{v}_{0} / 10^{-7} \mathrm{M} \mathrm{s}^{-1}$} \\
\hline & Experimental & $\begin{array}{c}\text { Ajuste não linear por } \\
\text { mínimos quadrados }\end{array}$ & $\begin{array}{c}\text { Lineweaver-Burk, } \\
\text { Equação } 2 \\
\end{array}$ & $\begin{array}{c}\text { Hanes, } \\
\text { Equação } 3\end{array}$ & $\begin{array}{c}\text { Eadie-Hofstee, } \\
\text { Equação } 4\end{array}$ & Equação 5 \\
\hline 1,2 & 1,7497 & 1,587 & 1,734 & 1,586 & 1,703 & 1,607 \\
\hline 2,4 & 2,6614 & 2,525 & 2,647 & 2,528 & 2,629 & 2,548 \\
\hline 3,6 & 3,0768 & 3,145 & 3,210 & 3,153 & 3,210 & 3,165 \\
\hline 4,8 & 3,4088 & 3,585 & 3,592 & 3,597 & 3,609 & 3,602 \\
\hline 6,0 & 3,7825 & 3,913 & 3,868 & 3,929 & 3,900 & 3,927 \\
\hline 7,2 & 4,0474 & 4,186 & 4,077 & 4,187 & 4,121 & 4,178 \\
\hline 8,4 & 4,4551 & 4,371 & 4,241 & 4,393 & 4,295 & 4,378 \\
\hline 10,8 & 4,8617 & 4,674 & 4,480 & 4,702 & 4,552 & 4,677 \\
\hline
\end{tabular}




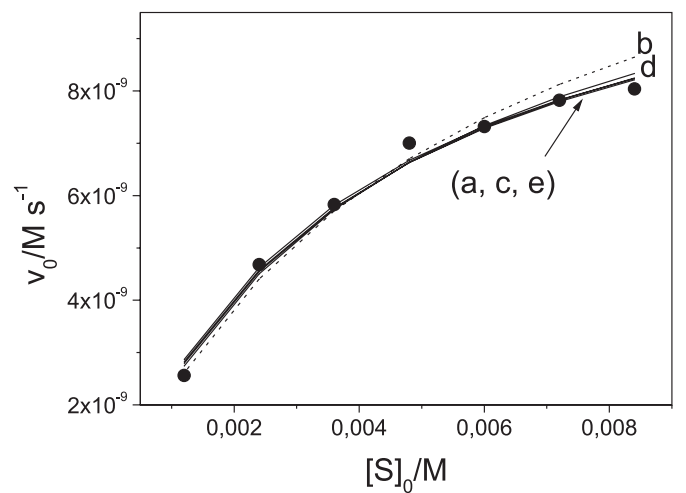

Figura 2. Valores experimentais $(\bullet)$ e calculados (linhas contínuas e pontilhadas) de $v_{0}$ para o conjunto de dados 2, usando a Equação 1 e os parâmetros obtidos por: a) ajuste não linear por mínimos quadrados, b) Equação 2, Lineweaver-Burk (linha pontilhada), c) Equação 3, Hanes, d) Equação 4, Eadie-Hofstee, e) Equação 5. Ver Tabela 4 para os valores numéricos

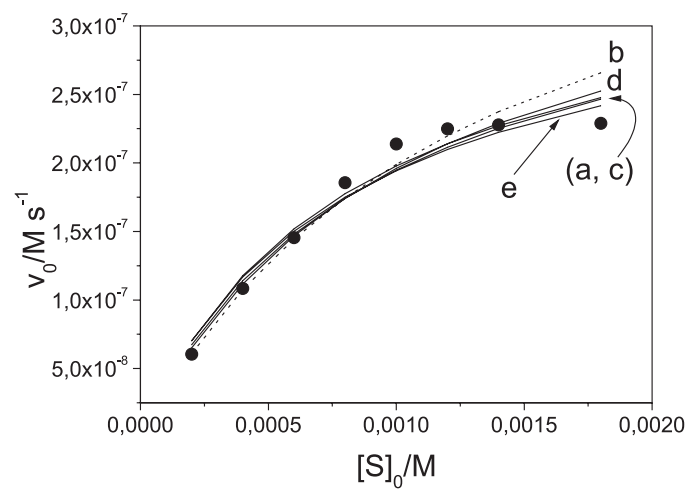

Figura 3. Valores experimentais $(\bullet)$ e calculados (linhas contínuas e pontilhadas) de $v_{0}$ para o conjunto de dados 3, usando a Equação 1 e os parâmetros obtidos por: a) ajuste não linear por mínimos quadrados, b) Equação 2, Lineweaver-Burk (linha pontilhada), c) Equação 3, Hanes, d) Equação 4, Eadie-Hofstee, e) Equação 5. Ver Tabela 5 para os valores numéricos

substituídos na Equação 1, produzem valores de $\mathrm{v}_{0}$ que se desviam significativamente dos valores experimentais, especialmente para as concentrações mais altas de substrato, conforme pode ser visto na Figura 1 e Tabela 2.

A fim de obtermos outros parâmetros de Michaelis-Menten que permitissem comparar o desempenho das Equações 2 a 5 em outras condições, aplicamos este mesmo procedimento para mais três conjuntos de dados: conjunto 2 - hidrólise de um fosfato de diarila por um complexo dinuclear de $\mathrm{Cu}$; conjunto 3 - hidrólise de um fosfato de diarila por um complexo dinuclear de $\mathrm{Ni}$; conjunto 4 - oxidação de um catecol substituído catalisada por um complexo dinuclear de $\mathrm{Cu}$.

Como pode ser visto na Tabela 3, as Equações 3 e 5 produzem, novamente, valores significativamente mais próximos daqueles obtidos por ajuste não linear por mínimos quadrados, do que os valores obtidos pelas Equações 2 e 4 . Vale notar que para os conjuntos de dados 2 e 3 os valores de $v_{0}$ calculados usando-se a Equação de Lineweaver-Burk (2) são maiores do que os valores calculados por ajuste não linear por mínimos quadrados (Tabelas 4 a 6 e Figuras 2 a 4), o que é uma situação diferente daquela mostrada na Tabela 2 e Figura 1. Por outro lado, os parâmetros de Michaelis-Menten obtidos pela Equação 2 para os conjuntos de dados 1 e 4 (Tabelas 2 e 3, respectivamente) produzem valores de $\mathrm{v}_{0}$ que são menores dos que os calculados por ajuste não linear por mínimos quadrados (Tabelas 2 e 6 e Figuras 1 e 4). Além disso, os dados experimentais que escolhemos são especialmente difícieis de serem ajustados, em especial

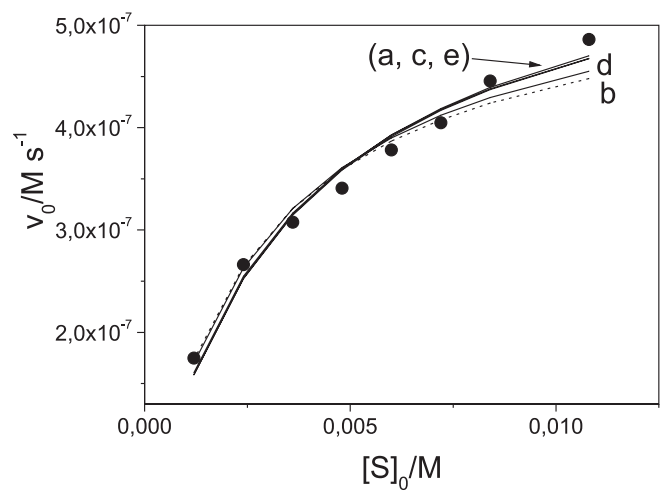

Figura 4. Valores experimentais $(\bullet)$ e calculados (linhas contínuas e pontilhadas) de $v_{0}$ para o conjunto de dados 4, usando a Equação 1 e os parâmetros obtidos por: a) ajuste não linear por mínimos quadrados, b) Equação 2, Lineweaver-Burk (linha pontilhada), c) Equação 3, Hanes, d) Equação 4, Eadie-Hofstee, e) Equação 5. Ver Tabela 6 para os valores numéricos

o conjunto de dados 3, para o qual mesmo o ajuste não linear por mínimos quadrados se afasta um pouco dos valores experimentais, conforme se pode ver na Figura 3 (ver também Tabelas 5 e 3).

\section{CONCLUSÕES}

Quatro equações lineares foram investigadas quanto a sua capacidade de produzir parâmetros de Michaelis-Menten confiáveis. Como resultado foi observado que duas delas, a Equação de Hanes (3) e a Equação apresentada aqui pela primeira vez (5), podem ser empregadas para determinar os parâmetros $V_{\max }$ e $K_{\mathrm{M}}$ através de um ajuste linear (regressão linear), uma vez que os resultados obtidos são bastante similares aos obtidos por ajuste não linear por mínimos quadrados. As equações escolhidas permitem uma abordagem didática simples e direta uma vez que os parâmetros de Michaelis-Menten são obtidos facilmente a partir dos coeficientes angular e linear das respectivas retas representadas por estas equações. A Equação 5 apresenta uma pequena vantagem de manipulação sobre a Equação 3 pelo fato dos valores de $V_{\max }$ e $-K_{\mathrm{M}}$ se apresentarem diretamente como os coeficientes angular e linear da reta. Por outro lado, embora mais confiável, o uso de um ajuste não linear por mínimos quadrados, requer pacotes de programas mais sofisticados e de manipulação mais complexa, exigindo a estimativa de valores iniciais e a realização de diversas iterações até que os valores dos parâmetros a serem obtidos não variem mais após novas iterações.

A comparação com os parâmetros obtidos por ajuste não linear por mínimos quadrados confirma também que a bem conhecida Equação de Lineweaver-Burk (2) e a Equação de Eadie-Hofstee (4) não produzem bons valores para os parâmetros de Michaelis-Menten, conforme já apontado por outros autores., ${ }^{2,4}$

\section{AGRADECIMENTOS}

Ao apoio financeiro do CNPq, CAPES, INCT-Catalysis e FAPERJ.

\section{REFERÊNCIAS}

1. Briggs, G. E.; Haldane, J. B. S.; Biochem. J. 1925, 19, 338.

2. Espenson, J. H.; Chemical Kinetics and Reaction Mechanisms, $2^{\text {nd }}$ ed.; McGraw-Hill: Nova Iorque, 2002.

3. Connors, K. A.; Chemical Kinetics. The Study of Reaction Rates in Solution, John Wiley: Nova Iorque, 1990.

4. Cornish-Bowden, A.; Fundamentals of Enzyme Kinetics, Portland Press: Londres, 1995. 
5. Michaelis, L.; Menten, M. L.; Biochem. Z. 1913, 49, 333.

6. Em ref. 2, p. 91.

7. Signorella, S.; Rompel, A.; Büldt-Karentzopoulos, K.; Krebs, B.; Pecoraro, V. L.; Tuchagues, J.-P.; Inorg. Chem. 2007, 46, 10864.

8. Lineweaver, H.; Burk, D.; J. Am. Chem. Soc. 1934, 56, 658

9. OriginLab Corporation, Origin 6.1; Northamptom, 2000.

10. Lotus Development Corporation; Lotus 1-2-3 Release 9 for Windows; 1998.
11. Hanes, C. S.; Biochem. J. 1932, 26, 1406

12. Eadie, G. S.; J. Biol. Chem. 1942, 146, 85.

13. Hofstee, B. H. J.; J. Biol. Chem., 1952, 199, 357.

14. Wilkinson, G. N.; Biochem. J. 1961, 80, 324

15. Dowd, J. E.; Riggs, D. S.; J. Biol. Chem. 1965, 240, 863.

16. Cornish-Bowden, A.; Eisenthal, R.; Biochem. J. 1974, 139, 721. 